\title{
Análise no Desempenho de Algoritmos de Aprendizagem Supervisionada na Classificação da Marcha em Parkinsonianos
}

\author{
Hugo A. Souza ${ }^{1}$, Marcelo Costa Oliveira ${ }^{1}$, \\ Leonardo Melo de Medeiros ${ }^{2}$
}

\author{
Instituto de Computação (IC), Universidade Federal de Alagoas (UFAL) \\ CEP 57500-970 - Maceió - AL - Brasil \\ Coordenação de Informática, Instituto Federal de Alagoas (IFAL) \\ CEP 57020-600 - Maceió - AL - Brasil \\ \{hasppgi@ic.ufal.br, oliveiramc@ic.ufal.br, leonardomelomedeiros@gmail.com\}
}

\begin{abstract}
The gait analysis has become an attractive and non-invasive quantitative mechanism that can aid in the detection and monitoring of Parkinson's disease patients. The extraction of characteristics is a task of paramount importance for the quality of the data to be used by the algorithms of Machine Learning, aiming as main objective the reduction in the dimensionality of the data in a classification process. This work evaluates the performance of supervised learning algorithms in the classification of human gait characteristics in PD patients.
\end{abstract}

Resumo. A análise da marcha tornou-se um mecanismo quantitativo atrativo e não invasivo que pode auxiliar na detecção e monitoramento de portadores da Doença de Parkinson. A extração de características é uma tarefa de suma importância para a qualidade dos dados a serem empregados pelos algoritmos de Aprendizagem de Máquina, visando como principal objetivo a redução na dimensionalidade dos dados em um processo de classificação. Este trabalho avalia o desempenho de algoritmos de aprendizagem supervisionada na classificação das características da marcha humana em portadores de DP.

\section{Introdução}

A Doença de Parkinson (DP) é a segunda doença neurodegenerativa mais prevalente em idosos, embora sua prevalência e incidência variem de acordo com a idade, sexo e raça/etnia [Tan 2013]. É uma doença crônica e degenerativa do sistema nervoso central, sendo caracterizada, principalmente, por distúrbios motores e disfunções posturais [Postuma 2012].

Estudos apontam que a prevalência aumenta com a idade, tendo estimativa de 5 a 26 casos a cada 100 mil pessoas por ano, sendo de aproximadamente $1 \%$ entre os indivíduos de 65 a 69 anos e, variando de $3 \%$ a 14,3\% entre os idosos acima de 85 anos [Willis 2010]. Os sinais cardinais (sinais clínicos mais comuns no processo inflamatório) mais comuns na DP incluem a presença de tremor em repouso, rigidez muscular, bradicinesia e instabilidade postural. O termo bradicinesia refere-se mais especificamente à lentidão no planejamento, iniciação e execução de atos motores voluntários e automáticos, associada à dificuldade na mudança de padrões motores, comprometendo diretamente o desempenho das atividades de vida diária destes pacientes [Kim et. al 2013]. Durante a locomoção o paciente com DP apresenta 
alterações hipocinéticas caracterizadas pela redução do comprimento dos passos, da velocidade e aumento do tempo de duplo apoio. Este sintoma pode ocorrer devido ao comprometimento na programação dos movimentos ou em sua execução [Stegemöller et. al 2012].

O diagnóstico de DP não é uma tarefa simples, pois sabe-se que há padrões de estágios do avanço da doença no organismo humano [Ângela, et al. 2016]. Porém, muitos pacientes não seguem esse progresso devido à heterogeneidade de manifestações que podem surgir [Postuma 2012]. Nos últimos anos, o diagnóstico da doença vem avançando graças aos investimentos realizados em pesquisas e a evolução dos aparatos computacionais. Utilizando a computação, pesquisadores estão ampliando seus conhecimentos sobre a DP, influenciados por informações cada vez mais precisas que vão desde o processamento de imagens neurológicas, detecção de modificações genéticas e análise de anomalias da marcha. A análise da marcha tornou-se um mecanismo quantitativo atrativo e não invasivo que pode auxiliar na detecção e monitoramento de portadores de DP. O diagnóstico está sendo ampliado por meio de recursos providos por áreas da computação, como a Aprendizagem de Máquina (AM) [Chang, Alban-Hidalgo and Hsu 2014], e na área de Processamento de Sinais [Daliri 2013].

A extração de características é uma tarefa de suma importância para a qualidade dos dados a serem empregados pelo algoritmo de AM, visando como principal objetivo a redução na dimensionalidade dos dados em um processo de classificação [Harrington 2012]. A partir da redução da dimensionalidade é possível identificar, principalmente, quais atributos são importantes e facilitar a visualização dos dados [Faceli 2011]. Para os dados relacionados à marcha humana, o propósito é detectar relevantes atributos que possam ajudar na identificação de fases do ciclo da marcha, como as fases de apoio e swing, cadência, comprimento da passada, velocidade, entre outras. Para tal, é preciso identificar e selecionar quais atributos são mais relevantes, assim como o método de classificação.

Visto que as técnicas de seleção de atributos já foram aplicadas em algoritmos de aprendizagem supervisionada na classificação de dados e mostraram um significativo desempenho na precisão (superior a 90\%) no diagnóstico de portadores de DP. Support Vector Machine (SVM) [Pant and Krishnan 2014], $k$-NN [Alkhatib et al. 2015] e Redes Neurais Artificiais (RNA) [Lee and Lim 2012], já foram utilizados em pesquisas com pacientes parkinsonianos aproveitando dados da marcha disponibilizados na base Physionet. Contudo, nenhum dos trabalhos publicados aplica mais de um algoritmo de AM manipulando atributos da base Physionet.

O objetivo deste trabalho é avaliar o desempenho de algoritmos de aprendizagem supervisionada na classificação dos padrões da marcha humana, sendo extraídas características como o total da Força Vertical de Reação do Solo (VGRF) sob os pés e o timestamp das fases de Apoio e Swing, em uma base de dados aberta.

\section{Metodologia}

O processo de classificação nesta pesquisa se deu seguindo as principais etapas de trabalhos. A base de dados que serviu para este trabalho está disponível na internet através do repositório aberto chamado Physionet [Physionet 2016]. Após a aquisição da base de dados, seis etapas foram desenvolvidas para a classificação (Figura 1): (i) 
Aquisição dos dados; (ii) Pré-processamento; (iii) Extração das Características; (iv) Seleção dos Atributos; (v) Aplicação dos Algoritmos de AM; e (vi) Validação.

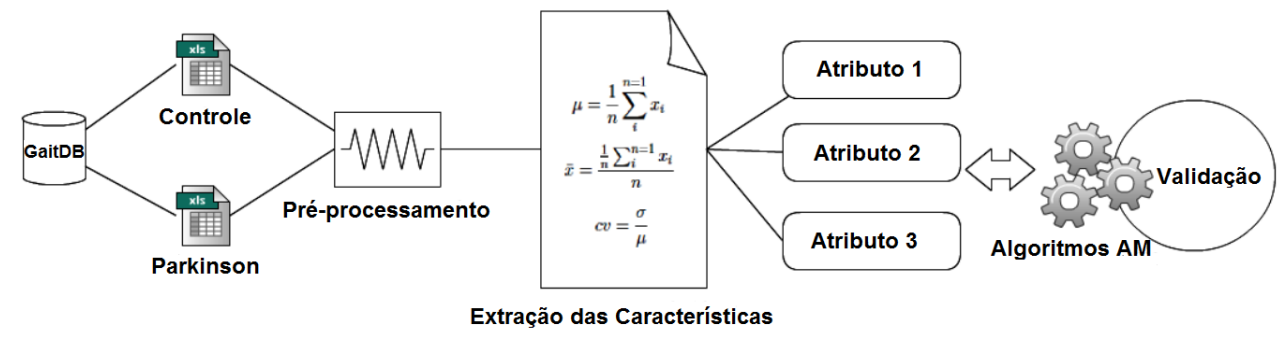

Figura 1. Etapas do processo de classificação.

A primeira etapa da classificação foi a aquisição dos dados. As amostras estão disponibilizadas no formato texto (TXT) e as variáveis estão dimensionadas por colunas. A primeira coluna de cada uma refere-se ao tempo (em segundos), e foi necessário extrair apenas uma como referência. Nas duas últimas colunas foram extraídos os dados do total da VGRF sob o pé direito e total da VGRF sob o pé esquerdo. Criadas duas planilhas eletrônicas (em formato XLS), a organização destas se deu em dois grupos: uma parkinsonianos e outra dos pacientes controle. A seleção das amostras foi restringida apenas as que possuem no mínimo 2 minutos de captura dos movimentos da perna direita e foi realizada de forma manual. Há pacientes com mais de uma amostra, mas só foi aproveitada apenas uma amostra por paciente. A partir dos critérios e passos já relatados, das 166 amostras disponíveis na base, foi reconhecido um quantitativo de 88 , destes 44 são pacientes controle e 44 parkinsonianos, dentre todas por grupo de pesquisa;

$\mathrm{Na}$ etapa seguinte, a pré-processamento, tem como trabalho principal nesta fase foi identificar as fases do ciclo da marcha por meio das variáveis escolhidas. As características extraídas dos ciclos da marcha foram as duas fases primárias: Apoio e Swing [Chang, Alban-Hidalgo and Hsu 2014]. Relacionando os dados do tempo e o total da VRGF em cada uma das pernas foi possível realizar o reconhecimento destas fases. Com base em estudo realizado na literatura [Pant and Krishnan 2014], para detectar o momento em que o paciente manteve a perna em apoio verificou-se quando o valor da VGRF é maior do que zero e relaciona com os respectivos tempos. Já para a fase de swing verifica-se quando o valor da VGRF é igual a zero e relaciona com seus tempos;

A etapa de extração das características tem como finalidade o reconhecimento dos padrões das características foram aplicadas funções de estatística descritiva e algumas específicas de processamento de sinais para obtenção de atributos a serem usados na classificação, já abordadas por trabalhos relacionados [Alkhatib et al. 2015, Dubey, Wadhwani and Wadhwani 2013, Guyon and Elisseeff 2003], são elas:

a) Média:

$$
\mu=\frac{1}{n} \sum_{n=1}^{i} x_{i}
$$

b) Mediana:

$$
\bar{x}=\frac{\frac{1}{n} \sum_{n=1}^{i} x_{i}}{n}
$$


c) Variância:

$$
\begin{gathered}
\sigma^{2}=\frac{\sum f_{i}\left(p m_{i}-\bar{x}\right)^{2}}{\sum f_{i}} \\
\sigma=\sqrt{\frac{\sum f_{i}\left(p m_{i}-\bar{x}\right)^{2}}{\sum f_{i}}}
\end{gathered}
$$

d) Desvio Padrão:

e) Coeficiente de Variação:

$$
c v=\frac{\sigma}{\mu}
$$

f) Curtose:

$$
k=\frac{\mu^{4}}{\sigma^{4}}
$$

g) Obliquidade:

$$
v=\frac{\left(x-\left(\mu_{3}\right)\right)}{\sigma^{3}}
$$

h) Interquartil:

$$
i q r=q_{3}-q_{1}
$$

Ainda, foram aproveitadas algumas funções comumente aplicadas em estudos relacionados a processamento de sinais que utilizaram a mesma base de dados [Alkhatib et al. 2015], já que melhor contemplam alterações fisiológicas do sinal eletromiográfico permitindo uma melhor análise, são elas:

i) Raiz Quadrada da Soma (RSS):

$$
R S S=\sqrt{\sum_{n=1}^{n} x n^{2}}
$$

Onde $n$ é um conjunto de valores e X é um vetor [Alkhatib et al. 2015].

j) Raiz do Valor Quadrático Médio (RMS):

$$
R M S=\sqrt{\frac{1}{n} \sum_{i=1}^{n} x_{i}^{2}}
$$

k) Poder

Densidade

de

$$
P D S=\int_{-\infty}^{+\infty} f_{x}(\tau) e^{j 2 \pi \tau} d \tau
$$

Sinal (PDS):

A equação da PDS é baseada na transformada de Fourier, onde $\tau$ é o tempo de observação, $f$ é a frequência e d $\tau$ é a função impulso. Já a Relação de Potência Pico-aMédia (RPP) nada mais é que a relação entre picos dos sinais sobre RMS.

$$
\begin{aligned}
& \text { 1) Relação de de } \\
& \text { Potência Pico-a- } \\
& \text { Média (RPP): }
\end{aligned}
$$

Duas funções geralmente usadas para analisar a potência espectral do sinal foram utilizadas. Sendo $\mathrm{P}_{\mathrm{i}}$ a $i$-ésima linha do espectro da potência e $M$ é o comprimento 
da frequência (essas duas variáveis fornecem algumas informações básicas sobre o espectro o sinal e suas mudanças em função do tempo):
m) Média da
Frequência do
Sinal (MNF):
n) Mediana
Frequência
Sinal (MDF):

$$
M N F=\frac{\sum_{i=1}^{M} f_{i} P_{i}}{\sum_{i=1}^{M} P_{i}}
$$

Reconhecida a necessidade de selecionar atributos mais importantes no contexto dos tipos de características extraídas e a possibilidade de eliminar possíveis redundâncias foi empregada uma técnica específica de FS. A técnica escolhida foi a Wrapper por ser de fácil entendimento e aplicação. Por meio da Wrapper é possível avaliar atributos multivariados com o método Forward/Backward. Ao aplicar esse método sob um conjunto vazio são adicionados atributos progressivamente originando subconjuntos, obtendo e avaliando um índice de desempenho para classificação [Guyon and Elisseeff 2003].

Segundo [Guyon and Elisseeff 2003], para $k-N N$ a seleção mais adequada é a Forward. Neste trabalho o método foi usado da seguinte forma, sendo n a quantidade de tipos de atributos, e levando em conta que no trabalho foram extraídos 14 tipos diferentes, a seleção foi gradativamente aplicando os atributos ao algoritmo $k-N N$. Lembrando que a seleção foi por subconjunto de atributos por perna sob grupos de parkinsonianos e controle. Para Árvores de Decisão os tributos também foram selecionados desta forma visto que houve sucesso no uso [Zhang et al. 2014]. Para os algoritmos SVM e RNA foi aplicado de forma Backward, conforme recomendação de [Guyon and Elisseeff 2003]. A partir de n, que representa a quantidade de tipos de atributos a seleção, foi reduzindo os atributos de 14 para apenas um. Sempre aplicando aos algoritmos de classificação e os validando em seguida. Os algoritmos de Aprendizagem Supervisionada presentes na pesquisa formam um total de quatro e as implementações estiveram de acordo com as principais referências encontradas. Por dez vezes os seguintes algoritmos foram aplicados: $k-N N$; Árvore de Decisão; RNA; e SVM;

A técnica holdout foi uma das aplicadas neste trabalho na fase de validação. Esse método se mostrou o mais simples de ser empregado e, conforme visto na literatura [Faceli 2011], pouco apresenta problemas tanto em grande quantidade de dados tampouco em menores. Segundo [Faceli 2011], nesse método o recomendado é reservar um subconjunto de $1 / 3$ para validação e o restante $2 / 3$ para treinamento. Outra técnica foi a $k$-fold, na qual foi aplicado em uma proporção de dez subconjuntos de dados (10-fold) mutuamente exclusivos. A partir disto, um subconjunto é usado para teste e os k-1 restantes são para estimação dos parâmetros. Este processo foi realizado o número de vezes da quantidade de subconjuntos distintos, ou seja, dez vezes alternando de forma circular o subconjunto de teste.

Mais uma utilizada foi a validação leave-one-out, que nada mais é que um caso específico do $k$-fold, com $k$ igual ao número total de dados $n$. Ao obter a matriz de confusão, foi possível calcular as seguintes medidas de desempenho: precisão, sensibilidade, especificidade e acurácia. A matriz é de extrema relevância ao trabalho, 
pois com a sensibilidade permitiu avaliar a classificação correta dos doentes e a especificidade permitiu avaliar a classificação correta dos sadios.

Tendo os resultados das classificações realizadas das fases Apoio e Swing em um das pernas e das duas pernas com cada algoritmo sob as três validações foram obtidas as médias e AUC da acurácia. Também, a partir da geração da sensibilidade e especificidade foram criadas as curvas ROC a fim de demonstrar visualmente uma comparação do desempenho dos classificadores.

\section{Resultados e discussão}

Na classificação realizada sob os dados da perna direita na fase Apoio o algoritmo que melhor classificou foi a SVM sob a validação $k$-fold, quando alcançou $95,45 \%$ na acurácia e 97,77\% de precisão (Tabela 1). O bom desempenho com a SVM nessa classificação foi devido ao processo de indução com base nos dados de treinamento, na qual é constituída pelo princípio de minimização do risco empírico, ou seja, a minimização do erro no treinamento. Entretanto, a minimização do risco empírico nem sempre ocorre, principalmente em conjuntos de dados menores [Faceli 2011].

Um algoritmo com desempenho semelhante foi o $k-N N$ quando alcançou $95,45 \%$ em acurácia e 93,62\% na precisão. A acurácia teve o mesmo resultado com a SVM, mas a precisão foi $4,15 \%$ menor (Tabela 1). Contudo, usando a validação holdout foi algoritmo que teve a pior acurácia com $31,82 \%$. Ademais, outro algoritmo que obteve bom desempenho na classificação da fase Apoio sob a perna direita utilizando a técnica de validação $k$-fold foi a RNA. A acurácia foi apenas $1,14 \%$ inferior, resultou em 94,31\%, e a precisão foi igual, 97,77\%.

Tabela 1. Resultado dos algoritmos na classificação da fase Apoio e Swing da perna direita por validações (a) holdout, (b) k-fold e (c) leave-one-out.

\begin{tabular}{|c|c|c|c|c|c|c|}
\hline Ciclo & Algoritmo & & Precisão & Sensibilidade & Especificidade & Acurácia \\
\hline \multirow{12}{*}{$\begin{array}{l}\frac{0}{8} \\
\frac{2}{2}\end{array}$} & \multirow{3}{*}{ k-NN } & (a) & $48,39 \%$ & $53,57 \%$ & $44,83 \%$ & $31,82 \%$ \\
\hline & & (b) & $93,62 \%$ & $52,39 \%$ & $93,02 \%$ & $95,45 \%$ \\
\hline & & (c) & $86,67 \%$ & $51,32 \%$ & $86,05 \%$ & $86,36 \%$ \\
\hline & \multirow{3}{*}{ Árvore Decisão } & (a) & $38,71 \%$ & $37,50 \%$ & $51,28 \%$ & $36,36 \%$ \\
\hline & & (b) & $79,45 \%$ & $50,72 \%$ & $79,06 \%$ & $78,40 \%$ \\
\hline & & (c) & $77,78 \%$ & $50,72 \%$ & $77,27 \%$ & $78,41 \%$ \\
\hline & \multirow{3}{*}{ RNA } & (a) & $90,32 \%$ & $50,91 \%$ & $90,00 \%$ & $62,50 \%$ \\
\hline & & (b) & $97,77 \%$ & $53,01 \%$ & $97,50 \%$ & $94,31 \%$ \\
\hline & & (c) & $95,56 \%$ & $51,81 \%$ & $95,24 \%$ & $94,32 \%$ \\
\hline & \multirow{3}{*}{ SVM } & (a) & $74,19 \%$ & $45,10 \%$ & $77,78 \%$ & $57,95 \%$ \\
\hline & & (b) & $97,77 \%$ & $52,38 \%$ & $95,25 \%$ & $95,45 \%$ \\
\hline & & (c) & $91,11 \%$ & $51,90 \%$ & $90,48 \%$ & $89,77 \%$ \\
\hline
\end{tabular}

A Árvore de Decisão foi o classificador que apresentou menor precisão com $38,71 \%$ sob validação da técnica holdout, por conseguinte $59,06 \%$ menor do que a obtida pelo algoritmo SVM sob validação $k$-fold (Tabela 1). Não há trabalhos relacionados à base que aplicaram esse algoritmo. O resultado na classificação da classe 
Parkinson com a Árvore de Decisão sob validação holdout teve 36,36\% de acurácia, inferior se comparado aos demais algoritmos.

Para esse desempenho baixo com a Árvore de Decisão em relação aos demais se deve a um problema denominado underfitting. Esse problema pode acontecer quando o conjunto de treinamento é pequeno e não é significativo, ou seja, não apresenta casos que futuramente serão exercitados. Como é o caso do treinamento dos algoritmos com a técnica de validação holdout. Ainda, pode ocorrer também instabilidades devido a ruídos na base, produzindo grandes variações no resultado final. Também, pode acontecer que dois ou mais atributos em nós sejam classificados de forma similar e pequenas variações podem alterar o resultado, modificando todas as subárvores [Faceli 2011].

A RNA e a SVM apresentaram uma maior tendência a identificar pacientes Controle, já que a maioria dos resultados da especificidade na classificação com esses algoritmos foi maior que $90,00 \%$ (Tabela 1 ). Em trabalho publicado por [Kim et al. 2013] relata sobre a dificuldade dos pacientes no equilíbrio, comprimento do passo reduzido e tremor até em membros inferiores. Esses sintomas podem ser apresentados na captura dos dados e gerar, por exemplo, timestamp com padrão diferente (ou não) para os pacientes parkinsonianos. Apesar de serem fatores que influenciam na geração de dados que podem identificar as duas classes (Parkinson e Controle), há algoritmos de aprendizagem supervisionada que não conseguem distinguir instâncias (um ou mais atributos) de cada classe.

Diferente da fase Apoio, todos os algoritmos apresentaram desempenho tanto na acurácia como na precisão superiores a $60,00 \%$ para a fase Swing da perna direita. Dois classificadores alcançaram $100 \%$ de precisão: Árvore de Decisão e SVM. O $k$-NN sob duas diferentes validações ( $k$-fold e leave-one-out) obteve uma considerável acurácia em 97,73\% (Tabela 2). Ainda, todos os classificadores apresentaram um desempenho melhor também na classificação de pacientes Controle, a exemplo da Arvore de Decisão e a SVM que alcançaram $100 \%$ de especificidade.

Tabela 2. Resultado dos algoritmos na classificação da fase Swing da perna direita por validações (a) holdout, (b) k-fold e (c) leave-one-out.

\begin{tabular}{|c|c|c|c|c|c|c|}
\hline Ciclo & \multicolumn{2}{|l|}{ Algoritmo } & Precisão & Sensibilidade & Especificidade & Acurácia \\
\hline \multirow{12}{*}{$\cdot \frac{D}{\sum_{\infty}^{\infty}}$} & \multirow{3}{*}{ k-NN } & (a) & $93,54 \%$ & $49,15 \%$ & $93,75 \%$ & $95,16 \%$ \\
\hline & & (b) & $95,56 \%$ & $50,00 \%$ & $95,56 \%$ & $97,73 \%$ \\
\hline & & (c) & $95,56 \%$ & $50,00 \%$ & $95,56 \%$ & $97,73 \%$ \\
\hline & \multirow{3}{*}{ Árvore Decisão } & (a) & $100 \%$ & $50,82 \%$ & $100 \%$ & $69,32 \%$ \\
\hline & & (b) & $93,33 \%$ & $49,41 \%$ & $93,48 \%$ & $96,59 \%$ \\
\hline & & (c) & $93,33 \%$ & $49,41 \%$ & $93,48 \%$ & $96,59 \%$ \\
\hline & \multirow{3}{*}{ RNA } & (a) & $96,77 \%$ & $50,85 \%$ & $96,67 \%$ & $67,05 \%$ \\
\hline & & (b) & $95,56 \%$ & $50,00 \%$ & $95,56 \%$ & $97,73 \%$ \\
\hline & & (c) & $91,30 \%$ & $51,85 \%$ & $90,69 \%$ & $94,31 \%$ \\
\hline & \multirow{3}{*}{ SVM } & (a) & $100 \%$ & $56,36 \%$ & $100 \%$ & $62,50 \%$ \\
\hline & & (b) & $97,78 \%$ & $53,01 \%$ & $97,50 \%$ & $94,32 \%$ \\
\hline & & (c) & $97,78 \%$ & $53,66 \%$ & $97,44 \%$ & $93,18 \%$ \\
\hline
\end{tabular}


$\mathrm{Na}$ avaliação de desempenho dos algoritmos na classificação com dados extraídos das fases de Apoio e Swing em apenas um das pernas dos pacientes foi utilizada também a curva ROC. O $k$-NN foi o algoritmo que obteve melhor AUC com $0,789(\sigma=0,248)$, conforme pode ser visto na Tabela 3 .

Tabela 3. Desempenho na classificação das fases Apoio e Swing da perna direita com AUC e $\sigma$ de cada algoritmo.

\begin{tabular}{|l|l|}
\hline \multicolumn{2}{|c|}{ AUC } \\
\hline k-NN & $0,789 \pm 0,248$ \\
\hline Árvore Decisão & $0,677 \pm 0,222$ \\
\hline RNA & $0,522 \pm 0,161$ \\
\hline SVM & $0,667 \pm 0,162$ \\
\hline
\end{tabular}

Resultado superior ao obtido por [Alkhatib et al. 2015] que classificaram pisadas dos paciente aplicando esse mesmo classificador sob dados de uma perna extraídos da Physionet e, o desempenho sendo avaliado pela curva ROC alcançou AUC igual a 0,611. Esse resultado se deu com a soma das variáveis dos oito sensores implantados nos pés dos pacientes, e não apenas nos totais da VGRF utilizadas neste trabalho. Quando a curva ROC foi aplicada a Árvore de Decisão e a SVM, os valores da AUC foram respectivamente $0,677(\sigma=0,222)$ e $0,667(\sigma=0,162)$.

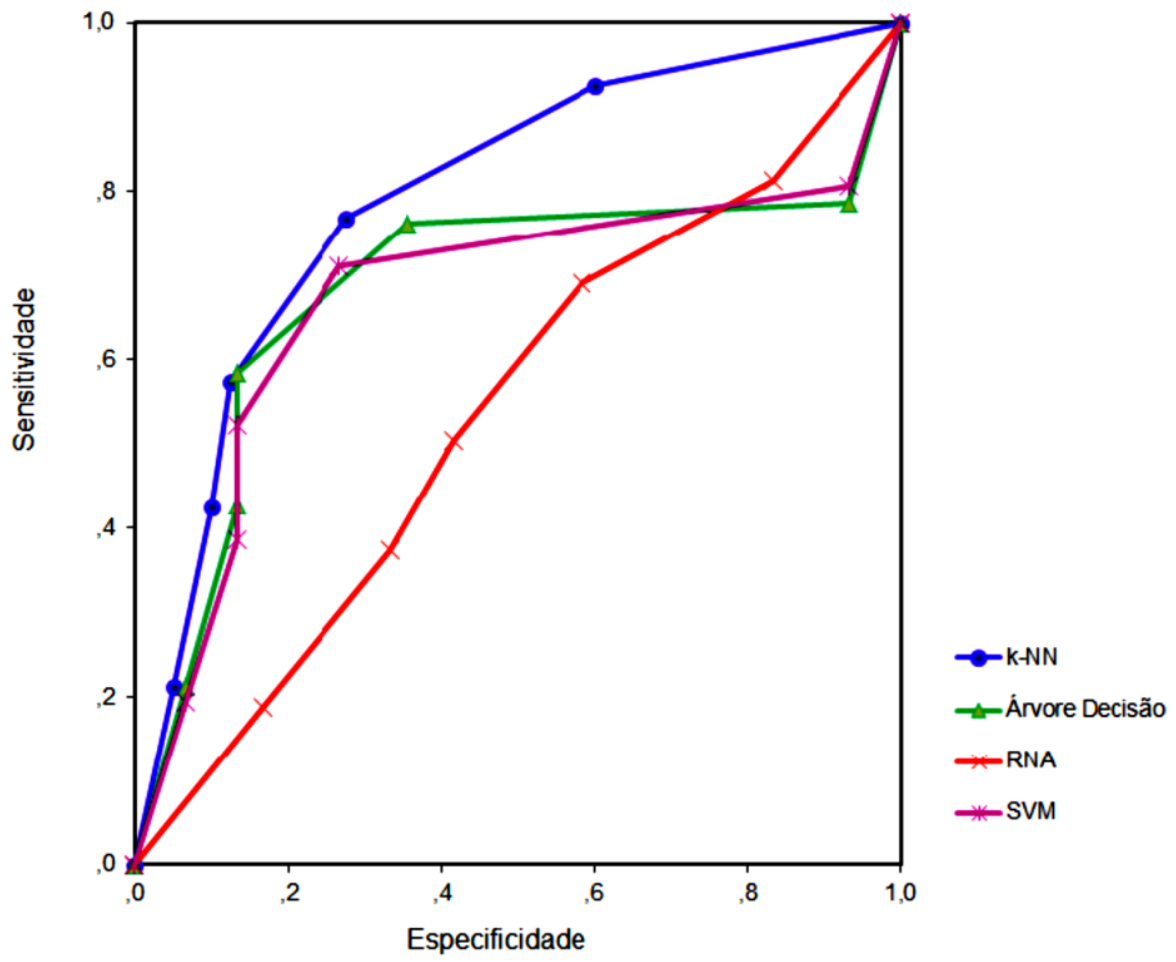

Figura 2. Avaliação desempenho dos algoritmos de aprendizagem supervisionada por meio da curva ROC na classificação das fases Apoio e Swing da perna direita.

Esses resultados foram inferiores ao alcançado por [Kamath 2015] quando classificou os dados das passadas de parkinsonianos (extraídos da Physionet). O autor 
aplicou entropia para classificar dados da perna direita e, na avaliação com a curva ROC, obteve AUC máxima de 0,885. Vale ressaltar que [Kamath, 2015] teve um maior conjunto de dados, pois usou de todos os sensores implantados nos pacientes que estão disponíveis na base Physionet. A RNA obteve um desempenho inferior entre todos nessa classificação com AUC igual a 0,522 $(\sigma=0,159)$.

\section{Conclusão}

Este trabalhou apresentou atributos que foram extraídos das fases de Apoio e Swing em portadores de DP e Controle, na qual podem influenciar no desempenho de algoritmos de aprendizagem supervisionada utilizados como classificadores. Com isso, buscou-se alcançar melhores resultados do que os que já foram alcançados em trabalhos relacionados. A SVM sob validação $k$-fold, foi o classificador que obteve melhor desempenho na fase Apoio sob a perna direita com 97,77\% de precisão. Já para a fase Swing, a maior precisão foi com os classificadores Árvores de Decisão e SVM quando alcançaram 100\% ambos sob validação holdout.

Embora a precisão na classificação dos dados tenha alcançado $100 \%$ com os algoritmos de aprendizagem supervisionada acredita-se que é possível aumentar os percentuais de sensibilidade, assim como também identificar mais características da marcha com a inserção de dois fatores que não foram utilizados nesta pesquisa: o aproveitamento de todas as variáveis extraídas dos sensores; e uso de todas as amostras disponíveis. Faz-se necessário também um estudo mais amplo sobre outras técnicas de seleção de atributos, como Embedded e Filtter, e mais algoritmos de AM. Com isso, espera-se que através dos resultados de mais classificadores sob mais atributos, analisado pelas respectivas técnicas de seleção, possa determinar resultados ainda melhores.

\section{Referências}

Alkhatib, R., Diab, M., Moslem, B., Corbier, C., El Badaoui, M. (2015). Gait-Ground Reaction Force Sensors Selection Based on ROC Curve Evaluation. Journal of Computer and Communications, 3(03), 13.

Chang, D., Alban-Hidalgo, M., Hsu, K. (2014). Diagnosing parkinson's disease from gait. Stanford.

Daliri, M. R. (2013). Chi-square distance kernel of the gaits for the diagnosis of Parkinson's disease. Biomedical Signal Processing and Control, 8(1), 66-70.

Dasgupta, H. (2015). An algorithm for stance and swing phase detection of human gait cycle. In Electronics and Communication Systems (ICECS), 2015 2nd International Conference, pages 447-450.

Dubey, M., Wadhwani, A. K., Wadhwani, S. (2013). Gait Based Vertical Ground Reaction Force Analysis for Parkinson's Disease Diagnosis Using Self Organizing Map. International Journal of Advanced Biological and Biomedical Research, 1(6), 624-636.

Faceli, K., Lorena, A. C., Gama, J., Carvalho, A. C. P. L. F. (2011). Inteligência Artificial: Uma abordagem de aprendizado de máquina. Rio de Janeiro: LTC, 2, 192. 
Fernandes, Â., Mendes, A., Rocha, N., \& Tavares, J. M. R. (2016). Cognitive predictors of balance in Parkinson's disease. Somatosensory \& motor research, 33(2), 67-71.

Geman, O., Ungurean, I., Popa, V., Turcu, C. O. and Găitan, N. C. (2012). Gait in Parkinson's Disease-signal processing and modeling. In 11th International Conference on development and application system, Suceava, Romania.

Guyon, I.; elisseeff, A. (2013). An introduction to variable and feature selection. Journal of machine learning research, 3, pages 1157-1182.

Harrington, P. (2012). Machine learning in action (Vol. 5). Greenwich, CT: Manning.

Kamath, C. (2015). A Novel Approach to Unravel Gait Dynamics Using Symbolic Analysis. Open Access Library Journal, 2(05), 1.

Kim, S. D., Allen, N. E., Canning, C. G., Fung, V. S. (2013). Postural instability in patients with Parkinson's disease. CNS drugs, 27(2), 97-112.

Lee, S. H., Lim, J. S. (2012). Parkinson's disease classification using gait characteristics and wavelet-based feature extraction. Expert Systems with Applications, 39(8), 73387344.

Pant, J. K., Krishnan, S. (2014). Foot gait time series estimation based on support vector machine. In Engineering in Medicine and Biology Society (EMBC), 2014 36th Annual International Conference of the IEEE, pages 6410-6413.

Physionet (2016). "Gait in Parkinson's Disease”. Homepage disponível em: $<$ https://physionet.org/physiobank/database/gaitpdb/>, Março.

Postuma, R. B. (2012). Identifying prodromal parkinson's disease: Pre-motor disorders in parkinson's disease. Movement Disorders. Wiley Online Library, 27(5): 617-626.

Salvatore, C., Cerasa, A., Castiglioni, I., Gallivanone, F., Augimeri, A., Lopez, M. and Quattrone, A. (2014). Machine learning on brain MRI data for differential diagnosis of Parkinson's disease and Progressive Supranuclear Palsy. Journal of Neuroscience Methods, 222, 230-237.

Stegemöller, E. L., Buckley, T. A., Pitsikoulis, C., Barthelemy, E., Roemmich, R., Hass, C. J. (2012). Postural instability and gait impairment during obstacle crossing in Parkinson's disease. Archives of physical medicine and rehabilitation, 93(4), 703-709.

Tan, L. C (2013). Epidemiology of parkinson's disease. Neurology Asia, 18(3): 231238.

Willis, A. W. (2010). Geographic and ethnic variation in parkinson disease: a population-based study of us medicare beneficiaries. Neuroepidemiology. Karger Publishers, 34(3): 143-151.

Zaknich, A. (2006). Principles of adaptive filters and self-learning systems. Springer Science \& Business Media.

Zhang, Y., Wang, S., Phillips, P., \& Ji, G. (2014). Binary PSO with mutation operator for feature selection using decision tree applied to spam detection. Knowledge-Based Systems, 64, 22-31. 